\title{
SOCIAL CRM E FINTECH: ANÁLISE DE PUBLICAÇÕES INTERNACIONAIS 2008-2018
}

\author{
Liliane Araújo ${ }^{1}$, Cristiana de Muylder ${ }^{1}$, João Ricardo Guerra ${ }^{1,2}$, Olaf Reinhold ${ }^{2}$ e Julio Viana ${ }^{2}$ \\ ${ }^{1}$ Universidade FUMEC - Programa de Doutorado e Mestrado em Administração, Belo Horizonte, Minas Gerais, Brasil \\ ${ }^{2}$ Social CRM Research Center, Leipzig, Saxônia, Alemanha
}

\begin{abstract}
RESUMO
Diante a fatores com globalização e ascensão tecnológica, proporcionando a intensa utilização de dispositivos móveis, surge o contexto capacitante para o estudo e desenvolvimento de SCRM (Social Customer Relationship Management). O SCRM tem por objetivo integrar a gestão de relacionamento com clientes às mídias sociais, de forma a buscar um melhor entendimento na forma de satisfação das necessidades dos stakeholders. Nesse sentido, o setor bancário percebe a necessidade de não apenas acompanhar esse movimento, mas sobretudo entender como novos playeres, sobretudo as Fintechs (Financial and Technology) passam a disputar esse mercado que é cada vez mais competitivo. Diante ao exposto, o trabalho em questão tem como objetivo avaliar as possibilidades de aplicação de SCRM nas Fintechs, e verificar como estão sendo estudados os assuntos que inter-relacionam esses temas. Na metodologia da pesquisa utilizou-se a análise cientométrica do software Vosviewer para identificar o vínculo entre as palavras-chave. A pesquisa bibliométrica foi realizada nas bases Elsevier, EBSCOhost e Inderscience, utilizando o construto Fintech, de janeiro de 2008 a dezembro de 2018. A análise permitiu identificar a mudança no mercado financeiro por meio de uma inovação disruptiva dos bancos digitais o que ocasionou uma revolução na forma de ofertar os produtos e serviços fazendários. Com a implementação da Fintech no setor financeiro, houve uma revolução na forma de captação de novos clientes por meio das redes sociais e internet, uma geração de consumidores com comportamento influenciado por essas redes, e com reflexo na oferta de produtos e serviços. Como sugestão para estudos futuros, propõe-se uma análise como uma forma comparativa para identificar a eficácia na utilização de $S C R M$ em Fintechs.
\end{abstract}

\section{PALAVRAS-CHAVE}

Social CRM, Fintech, Banco, Inter-Relacionamento, Revolução

\section{INTRODUÇÃOO}

O SCRM (Social Customer Relationship Management) é uma tecnologia que integra as ferramentas de mídia social e o gerenciamento de clientes para monitorar a análise de mercado. Espera-se que essa análise se torne mainstream para fornecer novas oportunidades para estratégias de colaboração, vendas e economia de custos conforme estudos de Greenberg, 2010, Banwo, 2018, Lee, \& Shin, 2018.

Com a utilização da internet e dispositivos móveis com funções diferentes, os consumidores passam a vislumbrar novas oportunidades diariamente. No setor financeiro, surge então uma nova forma de realizar as transações bancárias, um método mais democrático que envolve grande parte da população, através dos bancos digitais, as Fintechs (Financial and Technology), introduzindo no mercado bancário formas inovadoras dos mesmos serviços e produtos oferecidos pelos bancos tradicionais.

Os serviços Fintechs, Financial Startup e Online banking, segundo Chen, Li, Wu, \& Luo, 2017, Qi, Y., \& Xiao, 2018, fazem com que os bancos tradicionais busquem se atualizar às novas necessidades para garantir sua participação de mercado, conforme Reinhold \& Alt, 2009. Diante desse desafio, quais são as possibilidades de usar o SCRM nas Fintechs? Como estão sendo estudados os assuntos que inter-relacionam esses temas? Assim, este trabalho tem como objetivo elucidar e identificar quais são as discussões acerca dos constructos Fintechs e SCRM na academia internacional. 
Trata-se, portanto, de uma pesquisa descritiva, que utilizou artigos publicados nas bases Elsevier, EBSCOhost e Inderscience de janeiro de 2008 a dezembro de 2018. As bases selecionadas pelos autores foram adotadas pela relevância para a ciência, sendo compostas por periódicos de impacto científico em diversas áreas e países. O estudo pretende contribuir com finds sobre SCRM e Fintechs, promovendo e gerando novas discussões que possam atender às demandas do mercado financeiro e tecnológico. O setor financeiro passa portando a refletir sobre novos produtos e serviços focados em investimentos, alinhados entre o perfil e a demanda do consumidor por solução de problemas.

Nesse sentido, após a introdução do artigo realizada na sessão 1, tem-se a visão do novo mercado financeiro associada ao conceito de Fintech na parte 2, e na parte 3 será abordado o tema gerenciamento do relacionamento com clientes em mídia social. Nas seções 4 e 5 a apresentação da metodologia adotada para a pesquisa e os resultados, respectivamente, finalizando com as considerações finais no tópico 6.

\section{NOVO MERCADO FINANCEIRO: FINTECH}

A mobilidade tecnológica permitiu a expansão de serviços e produtos disponíveis para os clientes por meio de inovações, possibilitando um real estreitamento da relação entre o banco e consumidores, proporcionando ainda a entrada de negócios em mercados intermediários, de acordo com Faase, Helms, \& Spruit, 2011, Lak, \& Rezaeenour, 2017. Faase, Helms, \& Spruit, 2011 também acrescentam que as vantagens na adoção do banco digital podem ser verificadas: onipresença, imediatismo, localização, conectividade instantânea e funcionalidade proativa.

Uma característica importante na dinâmica de aceitação desse ambiente bancário, devido ao avanço da mobilidade, é a possiblidade dos usuários em acessar as informações financeiras a qualquer momento e em qualquer lugar de acordo com estudos de Faase, Helms, \& Spruit, 2011, Alt, Beck, \& Smits, 2018. Em contrapartida, Alt, Beck, \& Smits, 2018 afirmam que o volume de informações divulgadas no canal eletrônico de fidelização de clientes com o banco ficou restrito ao acesso de outras instituições, visto que atualmente, os usuários possuem relacionamento com vários bancos.

Assim, uma característica decisória da atração do cliente que escolhe adotar uma instituição específica é a proximidade, um envolvimento confiável entre usuários e instituições. Nesse sentido, para fortalecer a aliança e vincular a rede digital com os benefícios de realizar transações pela internet, de acordo com Hart, \& Gamal, 2012, Kim, Shin, \& Lee, 2009, Canaday, 2017, quanto mais próximo o cliente estiver da sua instituição, mais fácil será se conectar.

Na visão de Nguyen, \& Mutum, 2012, esse cenário suporta uma possível mudança no comportamento dos usuários bancários, que tendem a permanecer nesse nível de evolução de acordo com estudos de Há, Canedoli, Baur, \& Bick, 2012. A principal motivação para tal fato é que as novas gerações de consumidores estão cada vez mais afeitas às tecnologias e com menos tempo disponível para se comprometer com as rotinas bancárias.

Em resposta a esse novo ambiente, surge a expressão Fintech (Financial and Technology), para Li, Spigt, \& Swinkels, 2017, que é um novo serviço baseado em tecnologia, conforme comunhão de ideias entre Bergara, \& Ponce, 2017, Lehmkuhl, \& Jung, 2013, Greve, 2011, Mosadegh, 2011, Há, Canedoli, Baur, \& Bick, 2012, Mancini-Griffoli, 2018, Mai, Shan, Bai, Wang, \& Chiang, 2018, Alt, Beck, \& Smits, 2018, que pode ser usado por instituições financeiras e não financeiras de acordo com Bergara, \& Ponce, 2017, Ojeniyi, Alo, Oyetade, Ang, \& Sanusi, 2015. Nessa direção, estudos apontam para a possibilidade de compartilhamento de parcerias entre bancos e Fintechs, gerando opções para o consumidor acessar informações e serviços diferenciados, oferecendo novas opções financeiras e não financeiras (Bolchover, \& Symington, 2012, Li, Spigt, \& Swinkels, 2017, Lehmkuhl, \& Jung, 2013).

\section{GERENCIAMENTO DO RELACIONAMENTO COM CLIENTES EM MÍDIA SOCIAL}

Os sistemas de gerenciamento de relacionamento com clientes $(C R M)$, são um tipo de sistema de informações que permitem que as organizações entrem em contato com os clientes. Através da coleta, armazenagem e análise de dados dessa relação proporciona-se uma visão abrangente do mercado, de acordo com pesquisas de Gai, Qiu, \& Sun, 2018. 
O SCRM, ou CRM social, pode ser descrito como a expansão natural do CRM, com uma mudança na estratégia de marketing, citado por Leong, Tan, Xiao, Tan, \& Sun, 2017. Parte-se do pressuposto de envolvimento do cliente em uma abordagem colaborativa que busque a satisfação real das necessidades (De Muylder, Araujo, Isnard, Oliveira, Vasconcelos, 2018).

A definição mais usada de CRM social (SCRM) é de Greenberg, 2010, confluindo com os ensinamentos de (Greenberg, 2010, Ge, Feng, Gu, \& Zhang, 2017, Chen, Chen, \& Tsaur, 2016, Estrella-Ramon, Sánchez-Pérez, \& Swinnen, 2016, Gaughan, 2017, Heller Baird, \& Parasnis, 2011, Jiang, Ho, Yan, \& Tan, 2018, Pousttchi, \& Dehnert, 2018, Romānova, \& Kudinska, 2016). Entende-se, portanto, que o SCRM é "uma filosofia e uma estratégia de negócios, suportada por uma plataforma de tecnologia, regras de negócios, processos e características sociais, projetadas para envolver o cliente em uma conversa colaborativa, a fim de fornecer valor mutuamente benéfico em um ambiente de negócios confiável e transparente. Ou seja, é a resposta da empresa à própria conversa com o cliente". É uma atualização do gerenciamento original de relacionamento com o cliente, empregando mídias sociais ou mecanismos da Web 2.0.

Usando tais aplicativos de mídia social, o SCRM integra as atividades tradicionais de atendimento ao cliente, incluindo sistemas e tecnologias, com aplicativos emergentes de mídia social para envolver os clientes e outros atores em relações de colaboração, pela indicação de Montazemi, \& Qahri-Saremi, 2015.

\section{METODOLOGIA}

Utilizou-se a análise cientométrica do software Vosviewer para identificar o vínculo entre as palavras-chave que compõem os artigos das bases escolhidas pelos autores e a relação entre os autores e as citações presentes. A pesquisa bibliométrica foi realizada nas bases Elsevier, EBSCOhost, Inderscience, utilizando o construto Fintech de janeiro de 2008 a dezembro de 2018, identificando-se 479 artigos e utilizando as mesmas bases para o construto "Social CRM", encontrando 134 artigos no mesmo período.

A preferência pela base Elsevier deu-se por ser uma publicação renomada em âmbito internacional com periódicos e revistas de grande impacto na ciência. O caminho realizado na identificação dos 142 documentos encontrados na base Elsevier, foi confirmado de acordo com os critérios estabelecidos em pesquisa.

Finalizando a busca pelo construto Fintech, seguindo os critérios estabelecidos pelos autores, a base Inderscience colaborou com 34 documentos ao resultado. É possível inferir a baixa expressividade em publicação da temática quando comparada com as demais bases supracitadas, verificando-se uma oportunidade de desenvolvimento do tema. A importância da base no âmbito acadêmico internacional é relevante compondo-se de 436 periódicos em sua estrutura.

Seguindo os mesmos critérios apresentados no construto Fintech, pesquisou-se também por Social CRM, nas bases equivalentes, localizando 134 documentos no total. Posteriormente foi realizada uma busca na base EBSCOhost, mapeando 35 documentos. Já na base Elsevier, foram localizados 68 documentos utilizando os mesmos critérios estabelecidos. E por fim, pela base Inderscience foram encontrados 31 documentos com a busca da Social CRM no período estabelecido. Quanto aos critérios de inclusão e exclusão, estes são tratados e detalhados na Tabela 1.

A próxima seção reflete os resultados da pesquisa usando a ferramenta Vosviewer como alternativa a apresentar, quais as palavras-chave conseguem aproximar os dois temas. Optou-se pelas bases EBCOhost, Elsevier e Indersccience pela disponibilidade e facilidade de acesso aos pesquisadores, uma vez que se propõe a elucidar o assunto da Fintech e Social CRM na academia internacional. Além disso por se tratar de um tema referente a inovação, não seria possível identificar a evolução em períodos de pesquisa mais longos, por esse motivo houve o corte temporal de 11 anos. 
Tabela 1. Critérios de inclusão e exclusão

\begin{tabular}{l} 
Participantes: \\
Inclusão \\
Qualquer estudo que tivesse os termos Fintech ou Social CRM nas duas fases da pesquisa em qualquer seção do \\
documento. \\
Exclusão \\
Nenhuma restrição para exclusão foi aplicada. \\
\hline Resultados: \\
Artigos científicos sobre os dois temas chave. \\
Inclusão \\
Qualquer trabalho indexado nas bases pesquisadas que apresente estudos sobre Fintech ou Social CRM \\
contemplando aplicações tecnológicas e estudos de caso. \\
Exclusão \\
Qualquer trabalho que aborde assuntos que não tratem os termos pesquisados e apenas cite-os sem ser o foco do \\
artigo . \\
\hline Tipos de estudos: \\
Inclusão \\
Foram selecionados artigos que se enquadram nos seguintes filtros: \\
- Qualquer estudo do tipo journal article; \\
- Qualquer período de tempo; \\
- Trabalhos publicados em qualquer idioma. \\
Exclusão \\
Estudo em que os resultados não podem ser classificados; \\
Trabalhos duplicados; \\
Estudos que não abordam o tema necessário; \\
Publicações que não foram classificadas como journal article nas bases selecionadas.
\end{tabular}

Fonte: Elaborado pelos autores

\section{RESULTADOS}

Na pesquisa realizada pelos autores identificou-se 479 artigos no que tange ao construto Fintech e 134 Social CRM, totalizando 613 artigos que foram dispostos na ferramenta Vosviewer. Todavia, optou-se por trabalhar apenas por meio dos mapas de densidade e rede de conexão das palavras-chave e a relação entre elas, ou seja, buscou-se representar a união entre esses dois temas atuais, identificando nas pesquisas publicadas como a ferramenta da Social CRM está sendo utilizada pelas Fintechs.

Considerando a análise conjunta dos construtos Fintech e Social CRM, observa-se que essas palavras chave apareceram nos artigos pelo menos $1 \mathrm{vez}$, em todas as três bases pesquisadas. Por esse filtro, foi mapeado um total de 2040 itens, sendo divididos em 115 clusters, iniciando-se com 67 itens no primeiro grupo, seguido por $65,61,60$ e 55 itens por clusters.

Nota-se os termos em evidência como fintech, banks, techonological innovations, social media, social crm e techonological change:choices, demonstram como a referência da captação de dados por meio de Big data, mídia social, aprendizado de máquina trazem benefícios para as Fintechs. As aferições permitem o entendimento da necessidade de uma abordagem com os clientes de forma mais assertiva, o que transforma o cenário bancário que antes era dominado pelas instituições tradicionais, (Yanagawa, 2016, Brătăşanu, 2017; Canaday, 2017; Ge, Feng, Gu, \& Zhang, 2017; Bergara, \& Ponce, 2017; Mancini-Griffoli, (2018) Mai, Shan, Bai, Wang, \& Chiang, 2018).

Após a análise de mapa overlay, percebe-se que distância entre os itens se referem a aproximação de vínculos referentes a co-citação, ou seja, em que houve maiores co-citações entre as palavras-chave. Nota-se os mesmos termos em evidência, demonstrando como a aproximação dos temas tem sido elucidada na academia internacional.

Giannakis-Bompolis, \& Boutsouki (2014), em sua pesquisa realizada acerca do segmento bancário na Grécia, puderam identificar a ferramenta do CRM e Social CRM como influenciadores para novos clientes no sistema financeiro para adoção de produtos. Percebe-se que para o banco ou empresas do setor que utiliza desse mecanismo, pela adoção das tecnologias citadas, permite acesso à vanguarda do relacionamento com o 
consumidor, uma vez que os feedbacks capturados por meio das redes sociais possibilitam essa interação mais próxima.

Como oportunidade de se reinventar, os bancos tradicionais seguem os caminhos das Fintechs fazendo uso de evoluções tecnológicas como Bigdata, computação na nuvem, Lee \& Shin, (2018). Dessa forma, as instituições têm buscado ofertar um serviço personalizado e com maior eficácia para desenvolver uma estrutura de custo reduzido.

Vargha (2018), em seu estudo desenvolvido na Hungria, também corrobora com esse raciocínio, indicando que os bancos iniciaram na adoção do $C R M$, uma forma de se destacar em um mercado saturado. Aderindo a maneira inovadora das Fintechs por meio da leitura de hábitos, perfis, atividade e modo de utilização dos serviços pode-se ter uma maior visibilidade acerca dos problemas a serem solucionados.

Por meio da avaliação de densidade das palavras-chave, indicando os termos com maior representatividade dentro da base pesquisada, tem-se a consolidação dos objetivos do estudo em elucidar as possibilidades de utilização do Social CRM pelas Fintechs e as discussões acerca da temática na academia internacional. Os estudos confirmam essa mudança na estrutura financeira, vide os desafios das Fintechs, Gaughan, (2017) e as mudanças disruptivas no mercado, Canaday, (2017). O desenvolvimento em inovação tecnológica no setor bancário tradicional, Mancini-Griffoli, (2018), a adoção da inteligência artificial Banwo, (2018) pelo setor e a influência das redes sociais como forma de eficiência na oferta de produtos ao consumidor financeiro (Mai, Shan, Bai, Wang, X., \& Chiang, 2018), também caracterizam esse novo mercado.

Assim, as mídias sociais estão cada vez mais em evidência, pois são capazes de monitorar e controlar os hábitos, preferências, riquezas, Mancini-Griffoli, (2018). A posse desses dados torna-se ainda mais importante para o market-share das instituições e Fintechs, por esse motivo a expressiva relevância na aplicação de Social $C R M$ como uma ferramenta aliada à busca de efetividade e performance.

Nota-se pelo estudo realizado por Qi \& Xiao, (2018) na China, que a utilização de análise de dados por meio da rede social e internet, possibilita um panorama de riscos referentes a diversos assuntos, que poderão ser úteis tanto para o setor financeiro, como marketing e oferta de emprego. O comportamento do consumidor, tanto para os bancos tradicionais, como para as Fintechs sofre grande influência pelo uso da internet.

O interesse público é motivado por meio das ações impostas pela mídia social, Vasiljeva, \& Lukanova, (2016), logo a necessidade de investir em banco de dados, como o Big Data torna-se fundamental para o setor. Uma vez que a Fintech inovou no setor utilizando recursos diretos da mídia social, Brătăşanu, (2017), baseado na influência exercida por esse meio de comunicação com o consumidor, mostra-se uma nova solução frente aos desafios.

A aplicação do crowdsourcing, como forma de compartilhar conhecimentos por meio de criação de um produto ou ideia pelas Fintechs, permitiu o aprimoramento de produtos e soluções financeiras com melhor eficiência e aplicabilidade, redefinindo o modelo tradicional. Dessa forma identifica-se além de uma maior atração de clientes, o aumento direto da rentabilidade nos negócios (Canaday, 2017).

Diante do cenário apresentado, esta pesquisa identifica a utilização do Social CRM tanto pelas Fintechs como pelos bancos tradicionais, como permissiva para a análise do mercado financeiro de forma mais assertiva. Uma vez que o uso da mídia social e internet de forma geral são influenciadores do comportamento e mercado financeiro, como o valor das moedas criptografadas Bitcoin, Mai, Shan, Bai, Wang, \& Chiang, (2018), aceitação de crowdfunding (empréstimos P2P), Jiang, Ho, Yan, \& Tan, (2018), robô-advisor (consultores de investimentos), (Bergara, \& Ponce, 2017), o Social CRM aplicado às Fintechs tem se cunhado cada vez mais como uma característica necessária a essas instituições.

\section{CONSIDERAÇÕES FINAIS}

A pergunta norteadora deste trabalho elucida "quais são discussões acerca das Fintechs e o SCRM na academia internacional", como método utilizado para analisar as discussões no cenário acadêmico. Adotou-se, portanto, uma pesquisa descritiva por intermédio da análise bibliométrica, e foram identificados 613 artigos no total das buscas dos construtos Fintech e Social CRM, sendo 479 e 134 artigos, respectivamente, nas bases EBSCOhost, Elsevier e Inderscience, no período compreendido entre janeiro de 2008 a dezembro de 2018. 
A análise permitiu identificar a mudança no mercado financeiro por meio de uma inovação disruptiva dos bancos digitais, o que ocasionou uma revolução na forma de ofertar os produtos e serviços fazendários. Com a implementação da Fintech no setor financeiro, houve uma revolução na forma de captação de novos clientes por meio das redes sociais e internet. Surge então uma geração de consumidores com comportamento influenciado pelas redes sociais e com reflexo na oferta de produtos e serviços.

Compreende-se a identificação entre os construtos Fintech e Social CRM, na medida em que tal sintonia é primordial para identificar com maior assertividade o perfil, os hábitos e necessidades que os consumidores e usuários necessitam, de forma que a oferta dos seus serviços e produtos sejam mais eficientes, eficazes e rentáveis para todos stakeholders. Além disso, a evolução desse setor permitirá um equilíbrio no mercado financeiro, uma vez que os bancos tradicionais, antes detentores do market-share se veêm em uma situação desconfortável, diante à mudança disruptiva entre as gerações bancarizadas. Nessas circunstâncias, os prestadores de serviços tradicionais devem alinhar-se aos serviços ofertados pelas Fintechs como forma de sobrevivência no mercado e buscar uma maior adaptação para atender as novas exigências mercadológicas e comportamentais.

A pesquisa apresenta como limitação a restrição da análise em apenas três bases como EBSCOhost, Elsevier e Inderscience, apesar de ter sido uma escolha dos autores devido a conveniência e facilidade de acesso. Como sugestão para estudos futuros, propõe-se uma análise entre os gestores do setor financeiro tradicional e digital que tenham adotado e não tenham adotado as práticas de $S C R M$, como uma forma comparativa no intuito de identificar a eficácia na utilização do instrumento, sobretudo os resultados financeiros da aplicação.

Este estudo salienta a mudança ocorrida em organizações que foram obrigadas a examinar novos modelos e tecnologias de negócios, incluindo o rastreamento de mídias sociais. O SCRM vem a ser uma estratégia proativa para as Fintechs, que são a nova forma de negócios bancários com base no serviço online.

\section{AGRADECIMENTO}

FAPEMIG, FUMEC e ao Social CRM Research Center (Leipzig, Alemanha). Este estudo foi financiado em parte pelo CNPq - Código Financeiro 407907 / 2018-1 e um perfil de produção científica do CNPq Productivity Finan.

\section{REFERÊNCIAS}

Alt, R., Beck, R., \& Smits, M. T. (2018). FinTech and the transformation of the financial industry, https://link.springer.com/article/10.1007/s12525-018-0310-9

Banwo, A. (2018). Artificial intelligence and financial services: Regulatory tracking and change management. Journal of Securities Operations \& Custody, 10(4), 354-365.

Bergara, M., \& Ponce, J. (2017). ¿ Qué tan perturbadoras son las tecnologías financieras?. Monetaria, 39(2).

Bolchover, D., \& Symington, A. (2012). Getting closer to the customer. London, New York, Hong Kong, GenevaAlt, R., Beck, R., \& Smits, M. T.

Brătăşanu, V. (2017). Digital innovation the new paradigm for financial services industry. Theoretical \& Applied Economics, 24.

Canaday, T. (2017). Evolving a payments business to meet the demands of a distributed economy. Journal of Payments Strategy \& Systems, 11(1), 15-22.

Chen, M. C., Chen, S. S., Yeh, H. M., \& Tsaur, W. G. (2016). The key factors influencing internet finances services satisfaction: An empirical study in Taiwan. american journal of industrial and business management, 6(06), 748.

Chen, Z., Li, Y., Wu, Y., \& Luo, J. (2017). The transition from traditional banking to mobile internet finance: an organizational innovation perspective-a comparative study of Citibank and ICBC. Financial Innovation, 3(1), 12.

De Muylder, Cristiana F.; Araujo, L. A.; Isnard, P.; Oliveira, J. G.; Vasconcelos, I. M. P. Inovação no setor financeiro: Fintech e análise bibliométrica dos estudos realizados. In: XXI SEMEAD, 2018, São Paulo. XXI Semead. São Paulo: USP, 2018. v. 1. p. 1-14.

Estrella-Ramon, A., Sánchez-Pérez, M., \& Swinnen, G. (2016). How customers' offline experience affects the adoption of online banking. Internet Research, 26(5), 1072-1092. 
Faase, R., Helms, R., \& Spruit, M. (2011). Web 2.0 in the CRM domain: defining social CRM. International Journal of Electronic Customer Relationship Management, 5(1), 1-22.

Gaughan, M. (2017). Commentary: FinTech and the Liberation of the Community Reinvestment Act Marketplace. Cityscape, 19(2), 187-198.

Ge, R., Feng, J., Gu, B., \& Zhang, P. (2017). Predicting and deterring default with social media information in peer-to-peer lending. Journal of Management Information Systems, 34(2), 401-424.

Giannakis-Bompolis, C., \& Boutsouki, C. (2014). Customer relationship management in the era of social web and social customer: an investigation of customer engagement in the Greek retail banking sector. Procedia-Social and Behavioral Sciences, 148, 67-78.

Gai, K., Qiu, M., \& Sun, X. (2018). A survey on FinTech. Journal of Network and Computer Applications, 103, 262-273.

Greenberg, P. (2010). The impact of CRM 2.0 on customer insight. Journal of Business \& Industrial Marketing, 25(6), 410-419.

Greve, G. (2011). Social CRM - ganzheitliches Beziehungsmanagement mit Social Media. Marketing Review St. Gallen, 28(5), 16-21. doi:10.1007/s11621-011-0058-5

Ha, K. H., Canedoli, A., Baur, A. W., \& Bick, M. (2012). Mobile banking_insights on its increasing relevance and most common drivers of adoption. Electronic Markets, 22(4), 217-227.

Hart, S. W., \& Gamal, K. (2012). Social Customer Relationship Management - From Customer to Friend. EMICS 2012 Proceedings (pp. 75-88). Munich.

Heller Baird, C., \& Parasnis, G. (2011). From social media to social customer relationship management. Strategy \& leadership, 39(5), 30-37.

Kim, G., Shin, B., \& Lee, H. G. (2009). Understanding dynamics between initial trust and usage intentions of mobile banking. Information Systems Journal, 19(3), 283-311.

Lak, B., \& Rezaeenour, J. (2017). Effective Factors of Social Customer Knowledge Management (SCKM) in Organisations: Study of Electronic Service Providers in Iran. Journal of Information \& Knowledge Management, 16(02), 1750014.

Lee, I., \& Shin, Y. J. (2018). Fintech: Ecosystem, business models, investment decisions, and challenges. Business Horizons, 61(1), 35-46.

Lehmkuhl, T., \& Jung, R. (2013). Towards social CRM-scoping the concept and guiding research.

Leong, C., Tan, B., Xiao, X., Tan, F. T. C., \& Sun, Y. (2017). Nurturing a FinTech ecosystem: The case of a youth microloan startup in China. International Journal of Information Management, 37(2), 92-97.

Li, Y., Spigt, R., \& Swinkels, L. (2017). The impact of FinTech start-ups on incumbent retail banks' share prices. Financial Innovation, 3(1), 26.

Mai, F., Shan, Z., Bai, Q., Wang, X., \& Chiang, R. H. (2018). How does social media impact Bitcoin value? A test of the silent majority hypothesis. Journal of Management Information Systems, 35(1), 19-52.

Mancini-Griffoli, T., (2018) Banking on Change: new technologies promise to reshape the financial services industry.Finance \& Development.

Montazemi, A. R., \& Qahri-Saremi, H. (2015). Factors affecting adoption of online banking: A meta-analytic structural equation modeling study. Information \& Management, 52(2), 210-226.

Mosadegh, M. J. (2011). Using social network paradigm for developing a conceptual framework in CRM. Australian Journal of Business and Management Research, 1(4), 63-71.

Nguyen, B., \& Mutum, D. S. (2012). A review of customer relationship management: successes, advances, pitfalls and futures. Business Process Management Journal, 18(3), 400-419. doi:10.1108/14637151211232614

Ojeniyi, A., Alo, O. K., Oyetade, E. M., Ang, M. T., \& Sanusi, Y. K. (2015, April). Online banking user interface: Perception and attitude. In 2015 International Conference on Computer, Communications, and Control Technology (I4CT) (pp. 64-69). IEEE.

Pousttchi, K., \& Dehnert, M. (2018). Exploring the digitalization impact on consumer decision-making in retail banking. Electronic Markets, 28(3), 265-286.

Reinhold, O., \& Alt, R. (2009). Enhancing collaborative CRM with mobile technologies. BLED 2009 Proceedings, 36.

Vargha, Z. (2018). Performing a strategy's world: How redesigning customers made relationship banking possible. Long Range Planning, 51(3), 480-494.

Vasiljeva, T., \& Lukanova, K. (2016). Commercial Banks and Fintech Companies In The Digital Transformation: Challenges For The Future. Journal of Business Management, (11).

Wang, L., Li, M., Han, X., Zhou, R., Zheng, K., \& Liu, M. (2018). Improved Density Peak Clustering Algorithm Based on Choosing Strategy Automatically for Cut-off Distance and Cluster Centre. Tehnički vjesnik, 25(2), 536-545. 